\title{
PLANEJAMENTO ORIENTADO POR CENÁRIOS COM O USO DE MODELOS CAUSAIS LINEARES E DIAGRAMAS DE ESTOQUE E FLUXO
}

\section{SCENARIO DRIVING PLANNING WITH THE USE OF LINEAR CAUSAL MODELS AND STOCK AND FLOW DIAGRAMS}

\author{
Júlio César Bastos de Figueiredo \\ Professor titular \\ Escola Superior de Propaganda e Marketing - ESPM \\ Rua Joaquim Távora 1240, 2o Andar, CEP: 0415-013 \\ Fone: (011) 5081-8176; e-mail: jfigueiredo@espm.br
}

\begin{abstract}
RESUMO
Para que um determinado projeto de negócios possa ser avaliado, seus resultados devem ser antecipados e considerados de acordo com as possíveis mudanças no ambiente. $\mathrm{O}$ uso de modelos mentais e de cenários dinâmicos computacionais, durante $\mathrm{o}$ processo de planejamento, visa criar novas fontes de informação que sensibilizem os gestores e planejadores para as implicações de suas decisões sobre a organização. Neste trabalho, será apresentado um novo método de fácil implementação, e que oferece a capacidade de executar simulações contínuas de cenários a partir do levantamento das relações lineares de impacto e retardo entre as variáveis de um sistema. A existência hoje de diversas ferramentas para a modelagem computacional permitirá o uso extensivo desses conceitos em situações práticas de planejamento, tanto no ambiente profissional quanto no de aprendizado.
\end{abstract}

Palavras-chave: modelagem, simulação, cenários, dinâmica de sistemas, planejamento. 


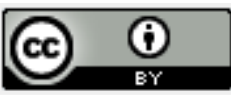

\begin{abstract}
A specific business project to be evaluated needs that your results be in advance considered, according to the possible changes in the environment. The use of mental and computational dynamic models aims to create new sources of information able to sensitize managers and planners alike to the dynamic implications of their decisions about the organization. In this work, it will be introduced a method which is easy to be implemented, capable of executing continuous scenario simulations by surveying linear casual relations among the variables that explain a system. Moreover, the existence of a number of system modeling tools will allow extensive application of the concepts used in the work to practical planning situations, both in the professional and educational settings.
\end{abstract}

Key-words: modeling, simulation, scenarios, system dynamics, planning.

\title{
1. INTRODUÇÃ̃
}

Cada vez mais pressionados pelo ritmo das mudanças do ambiente externo, e pela necessidade cada vez mais rápida de adaptação a estas mudanças, os gestores têm buscado formas mais estruturadas e objetivas para entender e lidar com a imprevisibilidade e a incerteza desses ambientes. Não é à toa, que desde a década de 70, com os trabalhos pioneiros de Pierre Wack na Shell, a abordagem estratégica através do uso de cenários tem ganhado força na área de planejamento (Van Der Heijden, 2004; Fahey, 1998; Georgantzas e Acar, 1995).

Para que um determinado projeto estratégico possa ser avaliado, seus resultados devem ser antecipados e considerados de acordo com as possíveis mudanças no ambiente. Para compreender essas mudanças, na maioria das vezes tenta-se a analisar o problema desmontando-o em partes para depois examinar essas partes e tentar compreender o todo. Todavia, a empresa e seu ambiente constituem-se em parte de um sistema. Um sistema é um conjunto de duas ou mais entidades ou elementos, cada qual tendo um papel de atuação sobre todo do sistema. O efeito de cada entidade depende dos efeitos causados por todas as demais entidades. Os sistemas apresentam propriedades que nenhuma de suas partes isoladamente poderia apresentar. Como consequência, um sistema não pode ser compreendido simplesmente dissecando-o, o que significa analisá-lo por partes. Para compreender completamente um sistema, deve-se identificar o todo, o contexto do qual o sistema faz parte, explicar o comportamento desse todo, e então explicar o comportamento do sistema em 


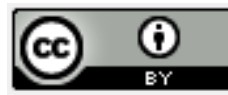

questão. Nos processos de planejamento nas empresas, a análise dos sistemas subjacentes deveria se tornar tão importante quanto à análise do comportamento das variáveis críticas do negócio, o que infelizmente não ocorre hoje, pelo menos em muitos casos.

\section{MODELOS MENTAIS}

É inegável a importância do pensamento sistêmico no processo de planejamento (Georgantzas e Acar, 1995, p. 300). Ao analisar uma empresa sob a ótica da teoria dos sistemas, vê-se que ela é composta de inúmeros elementos (que por si só podem ser considerados subsistemas). Esses elementos são integrados entre si e se relacionam funcionalmente objetivando a realização de um ou mais propósitos. A organização de um lado, recebe inputs do ambiente (energia, materiais, dados, etc.) e os transforma em outputs (produtos, materiais, informações, resíduos, etc.) que são então novamente devolvidos ao ambiente. A abordagem sistêmica do processo de planejamento tem como ponto de partida a construção dos modelos mentais que descrevem esses sistemas (Senge, 1994, p. 224). Tais modelos são representações das estruturas subjacentes aos sistemas organizacionais ou sociais que estão sendo analisados. Procura-se com eles examinar a inter-relação das forças estruturais em um contexto mais amplo que permita entendê-las como parte de um processo comum (Pidd, 1998, p. 118).

Em casos práticos, a construção e o trabalho com modelos mentais pressupõem o uso de alguma ferramenta de Mapeamento Cognitivo (Pidd, 1998, p. 139). O Mapeamento Cognitivo (Eden, 1988) faz parte de um conjunto de metodologias que dá corpo ao que se convencionou denominar Abordagem Soft da Pesquisa Operacional, voltada muito mais para a estruturação de problemas complexos, auxiliando no discernimento de soluções, do que para sua resolução propriamente dita. Essas técnicas de mapeamento procuram representar a percepção dos indivíduos envolvidos em uma situação problemática e fundamentam-se principalmente na Teoria dos Construtos Pessoais da área de psicologia (Kelly, 1955; Pidd, 1998, p.142).

Uma importante ferramenta de Mapeamento Cognitivo são os chamados Diagramas Causais (Sterman, 2000, p. 137). Os Diagramas Causais são diagramas que relacionam duas ou mais entidades de um sistema especificando que variações associadas a uma entidade irão gerar variações associadas à outra entidade. Ou seja, uma relação é dita causal se a ocorrência 
de um primeiro evento for condição necessária para a ocorrência de um segundo evento. Os diagramas causais são diagramas onde as entidades do sistema aparecem interligadas por setas representando as relações de causalidade. O sentido das setas indica o sentido da relação de causalidade. Além disso, cada seta pode possuir um sinal (+ ou -) indicando se as mudanças nas variáveis de causa e efeito têm o mesmo sentido (+) ou sentido contrário (-).

Como exemplo, considere o seguinte caso (adaptado de Georgantzas e Acar, 1995, p. 80): Uma empresa de prestação de serviços utiliza recursos de tecnologia de informação para disponibilizar para seus clientes, acesso estruturado e privilegiado a várias bases de dados de pesquisa na área de negócios. A capacidade de atendimento da empresa depende dos recursos disponíveis (pessoas, equipamentos, linhas de comunicação, etc.) que derivam de investimentos externos feitos. Entende-se que esta capacidade tem impacto direto na demanda gerada, sendo que a demanda aqui, corresponde às requisições de novos clientes e dos parceiros de conteúdo. Na medida em que as vendas da empresa crescem, isto impacta positivamente na imagem da empresa (dentro da região onde ela atua), gerando um impacto positivo na demanda. Por fim, o aumento da demanda irá gerar vendas, que por sua vez, irão gerar resultados que eventualmente serão convertidos em recursos. O diagrama causal resultante desse caso pode ser visto na Figura 1. Esse diagrama busca representar uma visão situacional das relações existentes e sua construção deve resultar em um diagrama claramente interpretado por todos os tomadores de decisão envolvidos em sua construção.

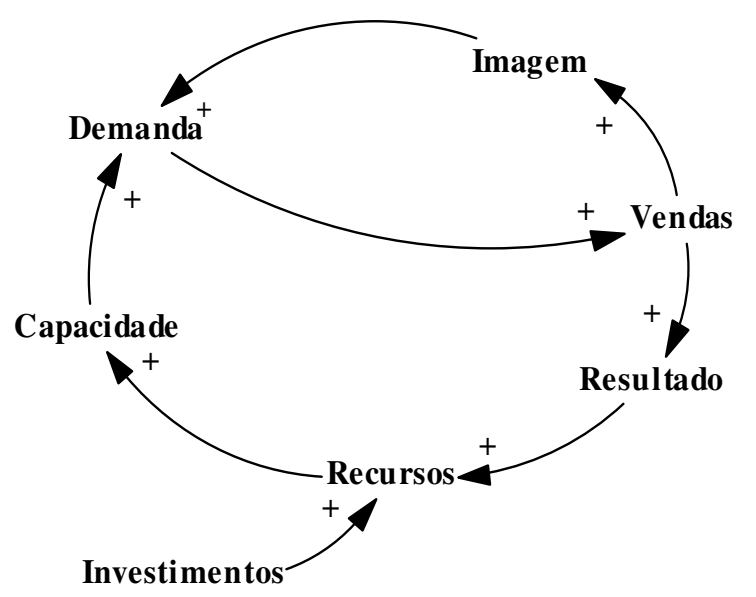

Figura 1: Exemplo de modelo mental (Diagrama Causal)

Fonte: Adaptado de Georgantzas e Acar (1995, p. 80) 


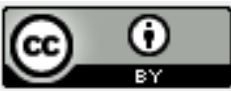

\section{MODELOS FORMAIS}

Apesar de sua importância para a representação dos modelos mentais dos participantes de um processo de planejamento, os diagramas causais falham em responder questões tais como: Que variável é mais decisiva e impactante para o funcionamento do sistema? Qual variável possui papel dominante para o sucesso de uma determinada estratégia? Quais os impactos das variações externas sobre o ambiente interno da empresa?

Para que dar respostas para essas questões, deve-se desenvolver um processo de modelagem formal. Modelos formais são basicamente modelos baseados em conceitos matemáticos e computacionais (Pidd, 1998, p. 192). A abordagem formal abre as portas para o desenvolvimento de capacidades de simulação, e assim, pode representar um bom ponto de partida para a modelagem estratégica através da simulação de cenários.

O uso prático e funcional das análises causais pressupõe o uso de modelagem computacional e simulação (Forrester, 1969; Roberts et al., 1983; Fahey e Randall, 1998; Sterman, 2000). Isso permitirá a avaliação dos efeitos das alterações nas variáveis externas e internas que afetam a situação de uma empresa. Torna-se possível a experimentação de alternativas e seu acompanhamento através da visualização do comportamento dessas variáveis. A partir dos modelos criados, podem-se definir vários cenários e testar seus impactos sobre o comportamento do sistema (Fahey e Randall, 1998, p. 157).

A construção de um modelo formal, que seja voltado para a simulação, pressupõe o conhecimento explícito das relações quantitativas entre as variáveis do modelo causal. Isto tem sido um empecilho para o uso mais efetivo de tais modelos nos processos de planejamento. A necessidade de especialistas em modelagem quantitativa e a dificuldade no levantamento preciso de todas as relações quantitativas consomem muito tempo e dinheiro. Uma solução seria o uso de uma formulação algébrica mais simples entre as relações de causa e efeito. Tal formulação deveria simplificar a construção do modelo formal sem, todavia, comprometer sobremaneira a análise dos resultados frente à realidade do negócio e de seu ambiente. Neste trabalho será proposto um método que modelagem formal que busca atender a esses requisitos. 


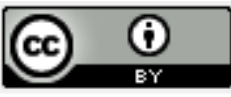

\section{OPERADORES CAUSAIS LINEARES}

David Heise, em seu livro sobre análise causal (Heise, 1975), formalizou a diagramação causal através de análises algébricas simplificadas. Heise argumentou que as transformações causais de eventos, operadas por estruturas materiais, não são nem raras nem impossíveis de serem decompostas em outras operações elementares. Segundo Heise, uma relação causal poderia ser efetuada por uma cadeia de operadores causais. Cada um desses operadores, responsável por transformar uma variação original (causa) em uma nova variação (efeito).

Por conveniência e simplicidade, Heise tratou os operadores causais como operadores lineares. Uma flecha causal direcionada de uma variável $A$ para uma variável $B$ expressaria uma função linear (ou transformação linear) de $A$, ou seja:

$$
A \stackrel{\alpha}{\longrightarrow} B \equiv B=\alpha \cdot A
$$

Ao diferenciar o lado direito da equação (1) obtém-se $d B=\alpha \cdot d A$. Isto significa que uma variação em $A$ automaticamente resultará em uma variação em $B$, escalonada pelo coeficiente escalar $\alpha$. Dependendo do fato de $\alpha$ ser maior ou menor que a unidade, a alteração em $B$ será relativamente maior ou menor do que a alteração em $A$. O sinal de $\alpha$ também indica se a alteração causada em $B$ tem o mesmo sentido, ou sentido oposto, da alteração causada em $A$.

Relações simultâneas de causalidade foram tratadas por Heise de forma aditiva:

$$
A \stackrel{\alpha}{\longrightarrow} B \stackrel{\beta}{\longleftarrow} C \equiv B=\alpha \cdot A+\beta \cdot C
$$

Note que a equação geral da reta é um caso particular da equação (2): $A \stackrel{\alpha}{\longrightarrow} B \stackrel{1}{\longleftarrow} \gamma \equiv B=\alpha \cdot A+\gamma$. A principal implicação da equação (2) é que os acúmulos dos efeitos resultantes de diferentes variações sobre uma variável poderão ser modelados de uma forma bastante simples e consistente em um diagrama de múltiplas relações. 
A formulação causal de Heise conduz ainda à seguinte relação transitiva:

$$
A \stackrel{\alpha}{\longrightarrow} B \stackrel{\beta}{\longrightarrow} C \equiv C=\alpha \cdot \beta \cdot A
$$

A equação (3) diz que uma alteração em $A$ irá gerar automaticamente uma alteração em $C$ como conseqüência de uma alteração em $B$. As relações causais diretas entre as variáveis $A$ e $B$, e entre $B$ e $C$, geram uma relação causal entre $A$ e $C$.

William Acar, em sua tese de doutorado (Acar, 1983), propôs uma extensão para a formulação original de Heise considerando a existência de atrasos físicos reais nos processos causais. Acar leva em conta um tempo de retardo para que ocorra uma transferência de variações entre os elementos de uma relação de causa e efeito, ou seja:

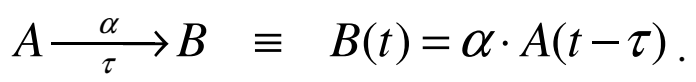

Acar também tratou as variáveis causais como níveis percentuais, ou seja, as variáveis causais registrariam, no tempo, a variação percentual acumulada das grandezas representadas. Assim, a relação vista na equação (4) poderia ser lida da seguinte forma: se a variável $A$ aumentar $\Delta \%$, então a variável $B$ irá aumentar proporcionalmente um valor $\alpha \cdot \Delta \%$, depois de um intervalo de tempo correspondente a $\tau$. Acar chamou este tipo de representação causal de Mapeamento de Situação Abrangente (Comprehensive Situation Mapping - CSM) (Acar, 1983; Georgantzas e Acar, 1994, p. 300). Os diagramas de CSM permitem construir um modelo simples de simulação, onde os impactos $\alpha$ são aplicados sobre as variações causais e transmitidos como efeito somente após os intervalos de tempo $\tau$, que correspondem aos atrasos presente nos ciclos (Georgantzas e Acar, 1994, p. 158).

Neste trabalho, será apresentado um novo método para a construção de modelos baseados na abordagem de Acar, com o uso da metodologia de Dinâmica de Sistemas. Esta formulação é de fácil implementação e oferece a capacidade de executar simulações contínuas de cenários a partir do levantamento das relações de impacto e retardo entre as variáveis de um sistema. A existência hoje de diversas ferramentas para a modelagem computacional permitirá o uso extensivo desses conceitos em situações práticas de planejamento, tanto no ambiente profissional quanto no de aprendizado. 


\section{DINÂMICA DE SISTEMAS}

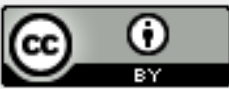

A Dinâmica de Sistemas é uma metodologia que busca estudar as estruturas de sistemas organizacionais ou sociais através da representação das relações causais entre seus elementos e através do estudo da evolução do sistema no tempo. Tem como objetivo principal explicar os comportamentos gerais dos sistemas, partindo dos padrões de comportamento entre as partes e das estruturas determinantes destes padrões (Forrester, 1961; Roberts et al., 1983; Pidd, 1998; Sterman, 2000).

Através de ferramentas e ambientes de simulação, permite ao pesquisador testar diferentes políticas e soluções para a operação do sistema, avaliando o impacto de suas decisões. Proporciona, por fim, um conjunto de instrumentos para compreensão e comunicação sobre os modelos da realidade (Pidd, 1998, p. 165). A Dinâmica de Sistemas assume que a análise de uma situação pode ser empreendida de um ponto de vista externo ao sistema, e que a estrutura e os processos dinâmicos podem ser recriados em diagramas com simbologia específica e modelos matemáticos apropriados.

Em Dinâmica de Sistemas a idéia fundamental é que o comportamento dinâmico de um sistema obedece a um princípio chamado de Princípio da Acumulação. Este princípio afirma que a resposta dinâmica de um sistema decorre da transição dos valores dos recursos acumulados em estoques. E que essas transições são controladas por fluxos de entrada e saída de recursos nos estoques. Ou seja, o comportamento dinâmico surge quando algo flui por algum meio. Esse princípio leva a uma forma particular de representação causal conhecida por Diagramas de Estoque e Fluxo (Sterman, 2000, p. 191).

Nestes diagramas, os estoques (ou níveis) são representados graficamente por retângulos. Eles representam caixas onde se acumula algum recurso no sistema. Os estoques são variáveis cujo valor (ou estado) depende dos fluxos. Os fluxos são representados por setas de traço duplo que são cortadas por um triângulo. Os fluxos representam o transporte dos recursos dentro do sistema. Os fluxos são vazões que são controladas por equações e por isto são representados por um ícone parecido com um cano com uma torneira. A Figura 2 mostra a representação básica de um estoque controlado por um fluxo em Dinâmica de Sistemas (a nuvem em uma das pontas da seta representa uma fonte de fluxo). 


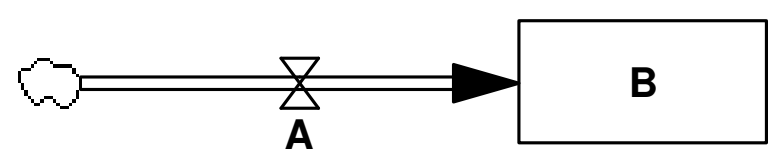

Figura 2: Estoque $(B)$ controlado por um Fluxo $(F)$.

Fonte: o autor

Na Figura 2, o valor do estoque $B$ é controlado pelo fluxo $F$. Esse controle pode ser expresso através de uma equação que representa a transição do valor de $B$ do tempo $t$ para o tempo $t+d t$. Essa transição é dada pela seguinte equação:

$$
B(t+d t)=B(t)+F(t) \cdot d t \Rightarrow F(t)=\frac{d B}{d t}
$$

Na modelagem com Diagramas de Estoque e Fluxo, tanto variáveis físicas como de informação podem fluir pelos fluxos se acumulando nos estoques. Dessa forma, considera-se aqui (como na abordagem de Acar) o caso onde os estoques registrarão os aumentos percentuais acumulados de cada uma das variáveis, e não os seus valores absolutos. Esse aumento percentual acumulado será sempre medido em relação a um patamar considerado inicial e os fluxos serão então fluxos de aumento percentual. Isso simplifica o processo de modelagem, pois elimina a necessidade de qualquer análise dimensional.

Na Figura 3, vê-se um estoque de percentual acumulado - $B$. Este estoque é afetado pelos valores de um segundo estoque - $A$. A relação causal entre esses dois estoques se dá através do fluxo $F$.

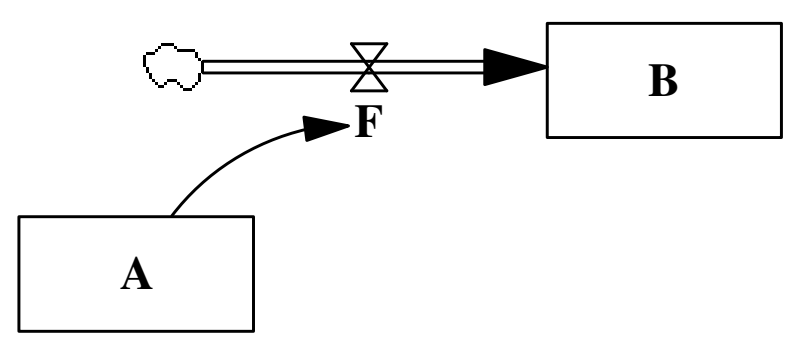

Figura 3: Relação causal entre dois estoques $A$ e $B$ por meio de um fluxo $F$. Fonte: o autor 
A relação causal entre o estoque $A$ e o fluxo $F$ é uma relação de transmissão de informação. Os Laços de Informação (representadas por setas de traço simples) ligam alguns elementos do sistema explicitando relações entre os mesmos. É importante observar que as relações de informação, diferente dos fluxos, não retiram ou colocam recursos nos estoques.

Usando a relação de transitividade da formulação de Heise (descrita na equação (3)) pode-se "ignorar" o fluxo $F$ e representar a relação causal entre $A$ e $B$. Definindo $i$ como sendo o coeficiente de impacto, que determina qual fração da variação será transmitida, e $r$ como sendo o tempo de retardo para que a transferência de impacto ocorra, podemos escrever:

$$
A(t) \underset{r}{\stackrel{i}{\longrightarrow}} B(t) \equiv B(t)=i \cdot A(t-r)
$$

Combinando agora a equação (6) com a equação (5) têm-se:

$$
F(t)=\frac{d}{d t} B(t)=i \cdot \frac{d}{d t} A(t-r)=\left.i \cdot \frac{d}{d t} A(t)\right|_{t=t-r}
$$

Portanto, o valor do estoque $B$ será controlado por um fluxo que corresponde à derivada com relação ao tempo do estoque $A$ no instante $t-r$ escalonada pelo coeficiente escalar $i$. Pode-se agora utilizar o princípio aditivo de Heise (equação (2)) para estender este conceito para um caso onde se têm múltiplos estoques. A Figura 4 apresenta um diagrama esquemático representando as funções de transferência entre vários estoques de acumulação percentual. As variáveis $i_{k}$ e $r_{k}$ correspondem, respectivamente, aos impactos e retardos entre as variações percentuais dos estoques. 

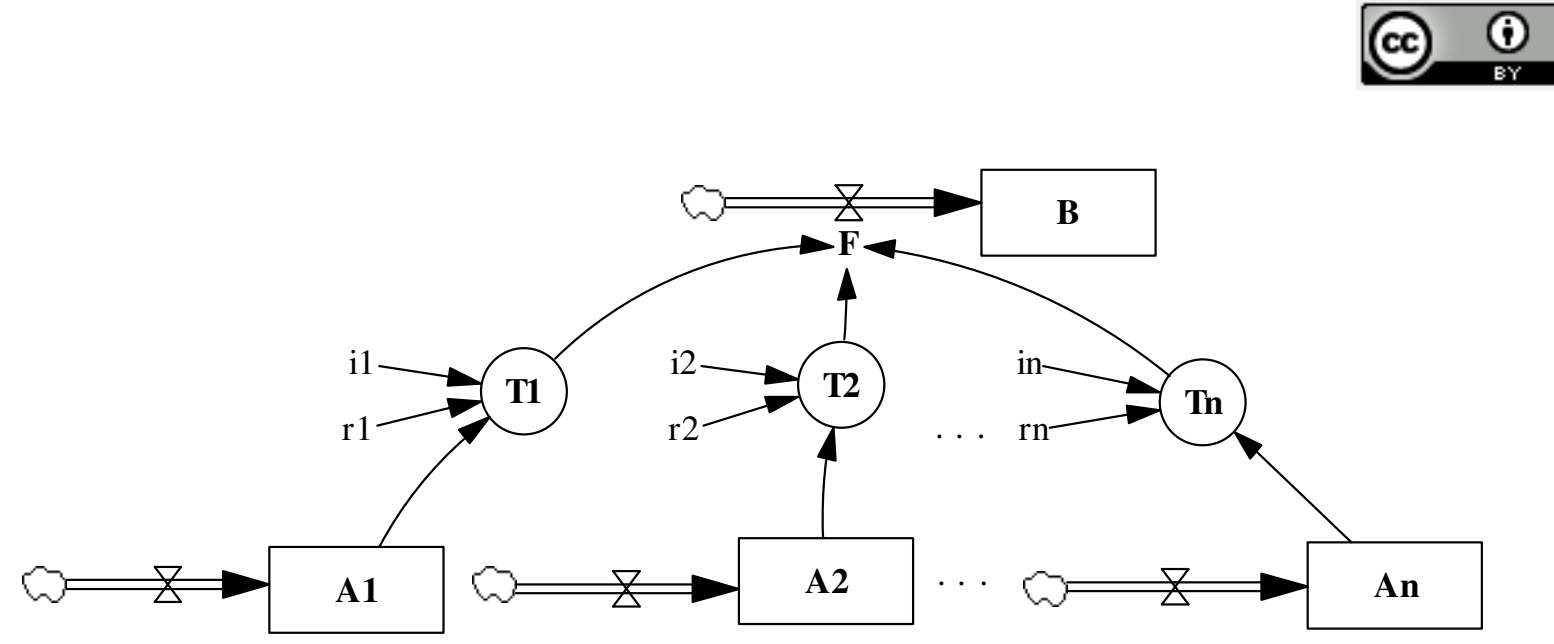

Figura 4: Diagrama esquemático representando as funções de transferência entre vários estoques de acumulação percentual. As variáveis $i_{k}$ e $r_{k}$ correspondem, respectivamente, aos impactos e retardos entre as variações percentuais dos estoques.

Fonte: o autor

Na Figura 4 as variáveis $T_{n}$ são funções auxiliares de transferência. Servem para calcular as derivadas com retardo no tempo. De forma explícita, as relações expostas na Figura 4 podem ser resumidas nas equações:

$$
\begin{aligned}
& B(t)=\int_{t_{0}}^{t} F(t) \cdot d t \\
& F(t)=\sum_{k=1}^{n} T_{k}(t) \\
& T_{k}(t)=\left.i_{k} \cdot \frac{d}{d t} A_{k}(t)\right|_{t=t-r_{k}}
\end{aligned}
$$

E assim, cada estoque $A_{k}$ pode ter seus fluxos modelados de maneira análoga.

\section{CENÁRIOS COMPUTACIONAIS}

O processo de modelagem em Dinâmica de Sistemas pressupõe a existência de ambientes computacionais próprios para a construção dos modelos simulados. Várias são hoje as plataformas que permitem a implementação de modelos com o uso dessa metodologia. A mais conhecida e utilizada destas plataformas é o software Vensim, da Ventana Systems (Vensim, 2004). Isso se deve principalmente ao fato de sua versão PLE (disponibilizada 


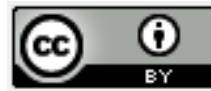

através da Internet) ser gratuita para fins acadêmicos e educacionais. Neste trabalho, os modelos apresentados foram desenvolvidos com o uso do software Vensim.

A construção de um modelo computacional que resolva o conjunto de equações (8) nem sempre é fácil. As ferramentas de simulação de Dinâmica de Sistemas felizmente já são desenhadas para implementar de maneira natural estruturas como as da Figura 4. Estão preparadas para integrar numericamente os fluxos para a obtenção dos valores dos estoques. Sendo assim, cabe ao modelista especificar as funções que representam cada variável auxiliar e deixar que o sistema se encarregue da solução numérica (Vensim, 2004).

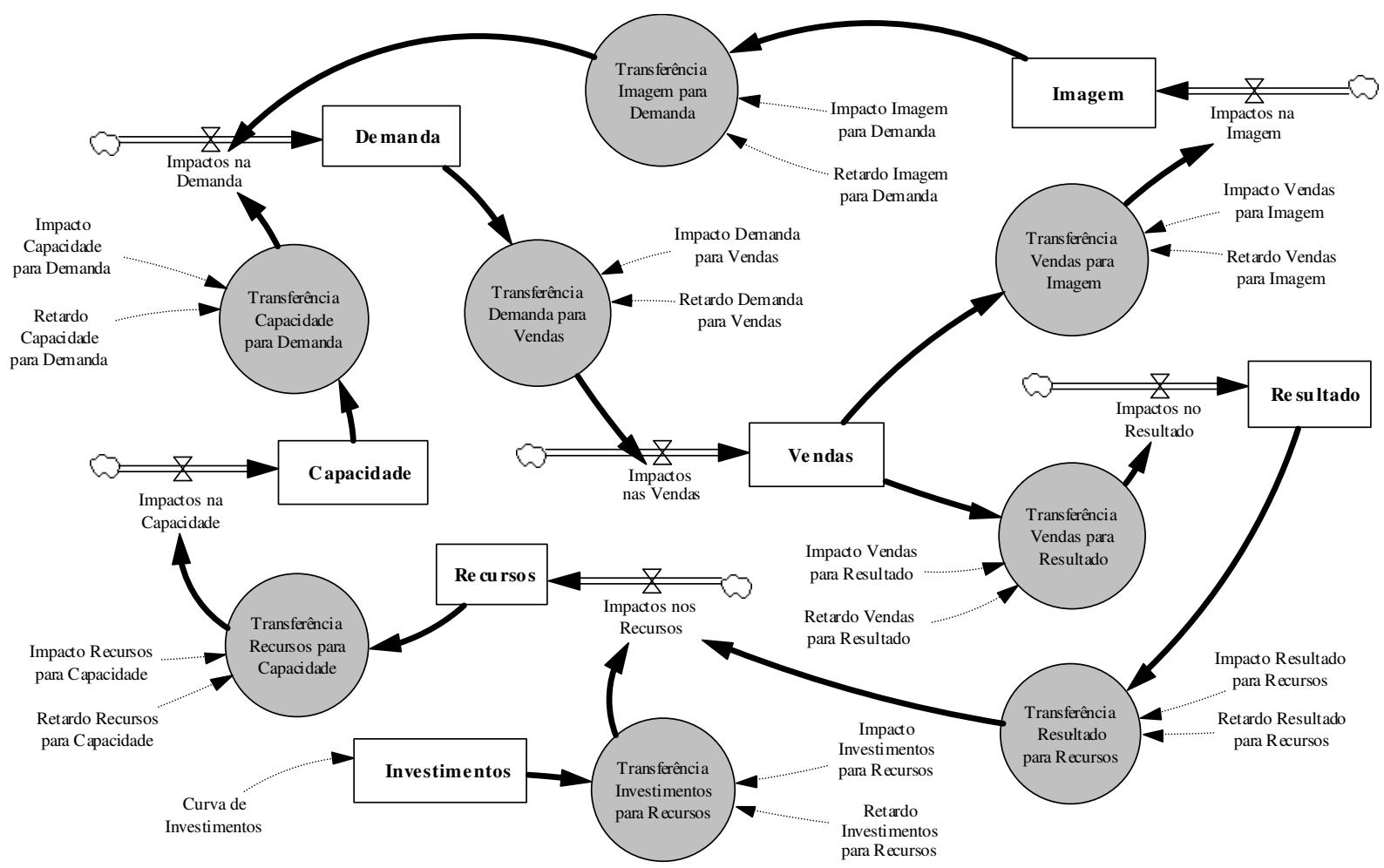

Figura 5: Representação do Diagrama de Estoques e Fluxos do modelo mental apresentado na Figura 1. Cada um dos estoques representa o acumulo de variação percentual da variável ao longo do tempo. Os fluxos desses acúmulos são controlados pelas funções de transferência calculadas de acordo com a equação (8).

Fonte: o autor

Na Figura 5 pode-se ver a representação do diagrama causal da Figura 1 utilizando a abordagem de estoques e fluxos (o código pode ser visto no Apêndice A). Note que cada variável é representada por um estoque e que este estoque registrará a variação percentual acumulada desta variável ao longo do tempo. Os fluxos que regulam estes estoques dependem dos estoques predecessores no diagrama causal. De acordo com a equação (8), cada fluxo é 


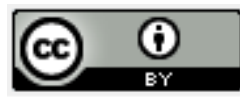

calculado através da soma das funções de transferências que chegam a ele e essas funções de transferência são definidas por um coeficiente de impacto multiplicado pela derivada temporal do estoque anterior em um instante de tempo retardado.

No diagrama da Figura 3, a variável A pode ser vista como uma variável independente ou exógena, e B como uma variável dependente ou endógena (isto é, cuja variação é determinada pelo sistema causal). São as alterações em A que direcionam o comportamento do sistema. Essas alterações são transportadas pela rede de relacionamentos e impactam a variável B. As variáveis do ambiente exógeno são as fontes de alteração para as variáveis do ambiente endógeno. O papel da modelagem de cenários é o de revelar e tornar compreensíveis os impactos operacionais das causalidades externas sobre o ambiente interno.

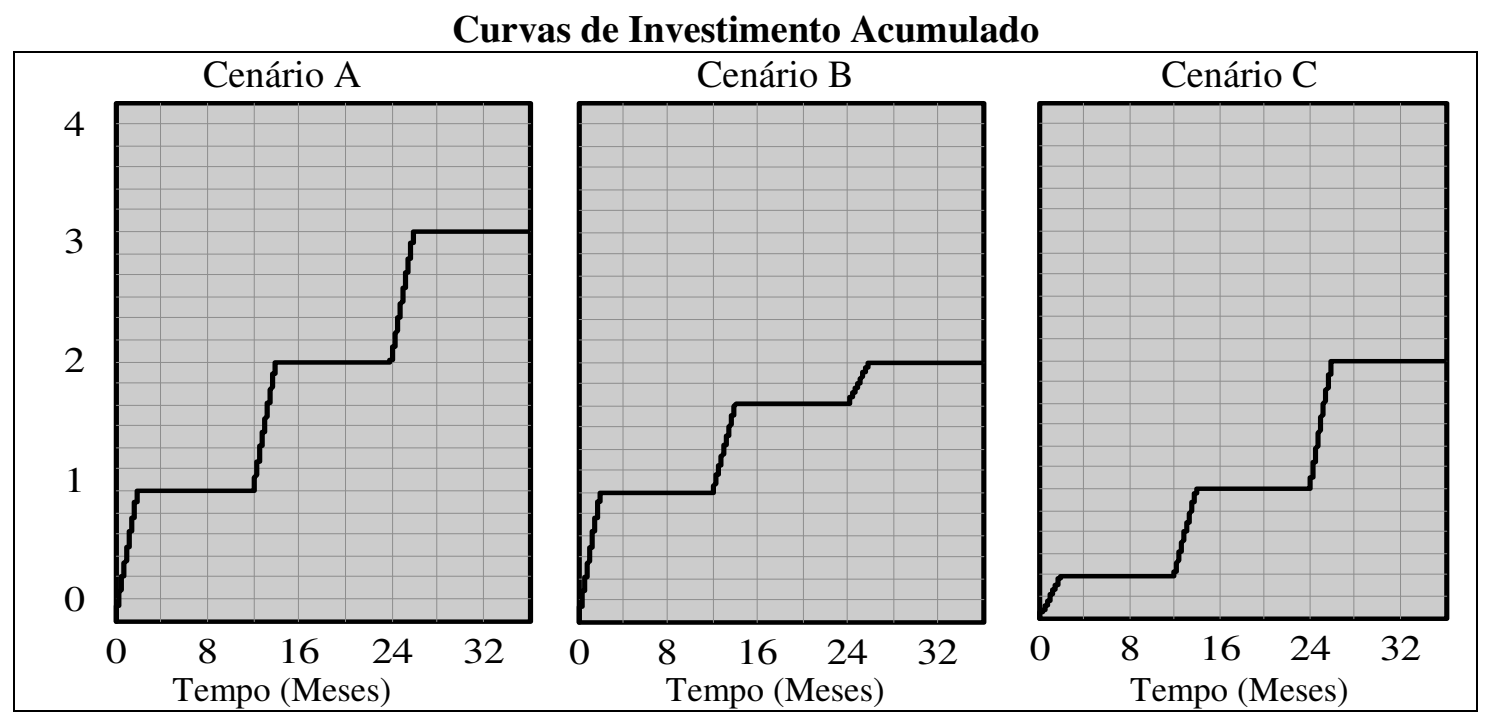

Figura 6: Três curvas de investimento acumulado correspondentes a três cenários possíveis de investimentos para o caso representado na Figura 5 (no eixo das ordenadas $1 \rightarrow$ $100 \%)$.

Fonte: o autor

No modelo da Figura 5, a única variável exógena considerada são os Investimentos recebidos pela empresa. Pode-se ver que esta variável depende de uma "Curva de Investimentos". Os formatos que essa curva pode assumir no tempo é que definirão os cenários possíveis de investimentos da empresa. Na figura (6) estão representadas (ao longo de 36 meses) três curvas de investimentos que, hipoteticamente, poderiam corresponder aos cenários previstos. No cenário A o nível de investimento cresce $100 \%$ no primeiro mês, mais 
$100 \%$ no décimo segundo mês e mais $100 \%$ no vigésimo quarto mês. No cenário $\mathrm{B}$, os aumentos ocorrem nos mesmos meses e correspondem a 100\%, $67 \%$ e $33 \%$ respectivamente. No cenário C, a sequência é de 33\%, 67\% e 100\%. O crescimento do aumento acumulado é sempre medido em relação ao patamar inicial.

Pode-se agora analisar o comportamento do sistema frente a estes cenários externos e determinar que tipo de influência tal comportamento tenha sobre as variáveis consideradas críticas para a saúde do negócio, como por exemplo, o Resultado. Pode-se também testar ajustes nas relações de impacto e retardo de forma a buscar minimizar os efeitos de algumas configurações ameaçadoras de cenário.

Para que isto possa ser feito, deve-se primeiro discutir quais são os valores de impacto e retardo que se acredita corresponderem a cada relação causal.

A Figura 7 mostra um painel que pode ser utilizado para discussão e testes desses valores (os retardos estão indicados em meses). Este painel possui um conjunto de chaves deslizantes representando cada um dos coeficientes de impacto e retardo. Estas chaves podem ser modificadas de tal forma a testar seus efeitos conjuntos sobre variáveis pré-selecionadas (no caso da Figura 7, foram escolhidas as variáveis: Recursos e Resultado). A idéia é que os tomadores de decisão discutam, baseados em suas experiências e nos dados levantados, os valores mais razoáveis para os impactos e retardos. Confrontando-os com as situações da realidade.

Caso se deseje observar o efeito das mudanças nos impactos e retardos em um contexto mais amplo, considerando mais variáveis, podem ser utilizados ambientes de simulação como o apresentado na Figura 8. Neste caso, podem ser observadas todas as implicações nas alterações dos impactos e dos retardos, simultaneamente sobre todas as variáveis, incluindo os fluxos de transmissão e as variáveis de transferência.

Considere agora o conjunto de valores de impacto e retardo exposto na Figura 7. Com este conjunto de valores construiu-se, para cada um dos cenários de investimentos (vistos na Figura 6) uma simulação de 36 meses. Escolheu-se a variável Resultado como variável de observação. Os resultados das simulações estão apresentados na Figura 9. Pode-se perceber claramente nessa figura que o cenário $\mathrm{C}$ gera um Resultado acumulado muito inferior aos conseguidos nos cenários A e B no período de 36 meses considerado. 

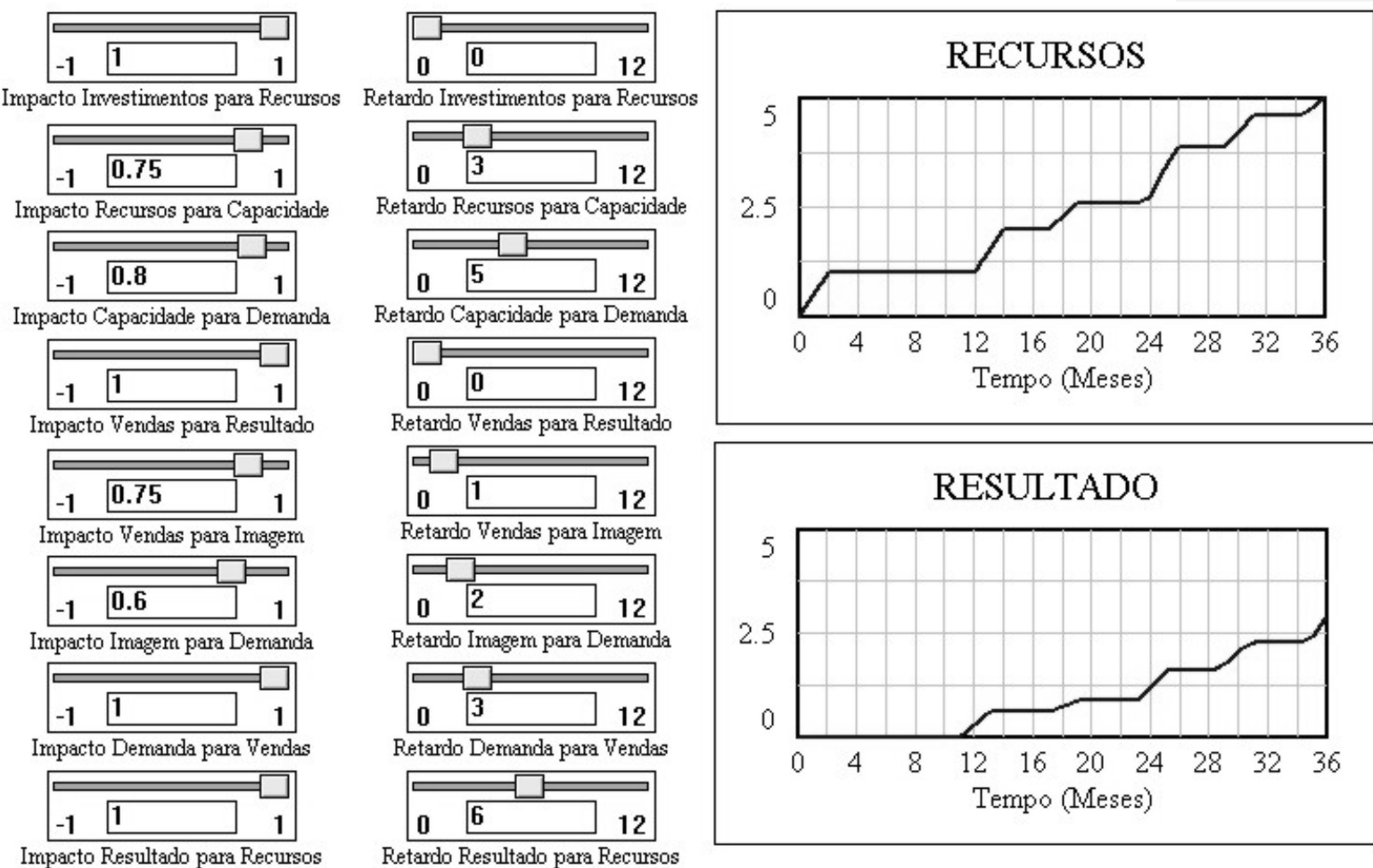

Retardo Vendas para Resultado
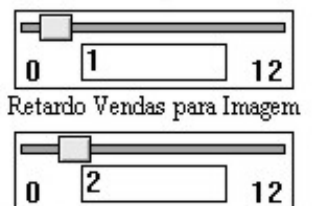

Retardo Imagem para Demanda

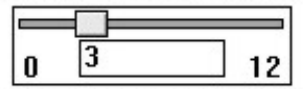

Retardo Demanda para Vendas

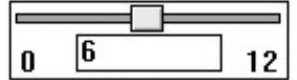

Retardo Resultado para Recursos

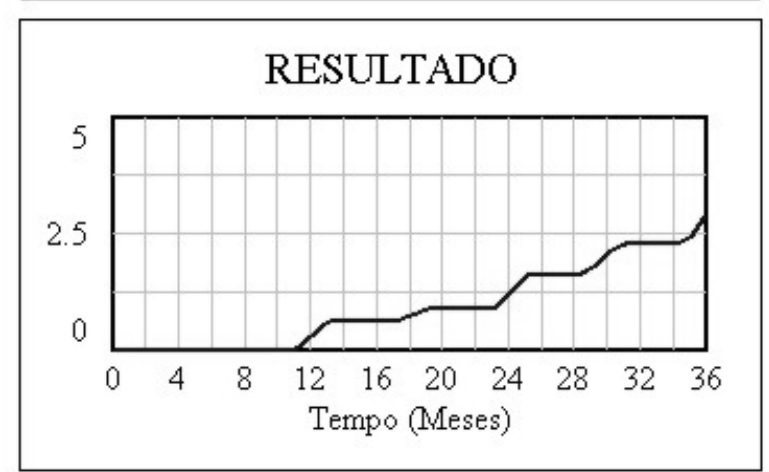

Figura 7: Painel de simulação para a discussão e seleção de valores das variáveis de impacto e retardo (medido em meses). A escolha pode ser feita testando o efeito das escolhas sobre as variáveis: Recursos e Resultado. O cenário de investimentos utilizado foi o cenário de A (Figura 6).

Fonte: o autor

Isso se explica tendo em vista o fato de que o cenário $\mathrm{C}$ é um cenário onde o volume inicial de investimento é muito menor que nos outros dois cenários, isso acaba inibindo o crescimento da capacidade de atendimento e, portanto, a geração de novas demandas. Isso retarda o ciclo de geração de vendas o que faz com que o crescimento acumulado do Resultado seja muito mais baixo no período quando comparado com os outros dois cenários.

Mas caso se confirme o cenário $\mathrm{C}$ como sendo o cenário de investimentos para os próximos 36 meses, o que poderia ser feito para minimizar o baixo retorno acumulado no final do período? Em primeiro lugar pode ser identificada uma inibição no crescimento da capacidade de atendimento, devido aos baixos níveis iniciais de financiamento. Logo, seria uma conjectura bastante razoável a de que um aumento na eficiência no processo de transformação dos Recursos para a Capacidade, bem como uma diminuição no prazo para que essa transformação ocorra, minimizaria os efeitos dos baixos níveis iniciais de financiamento. 


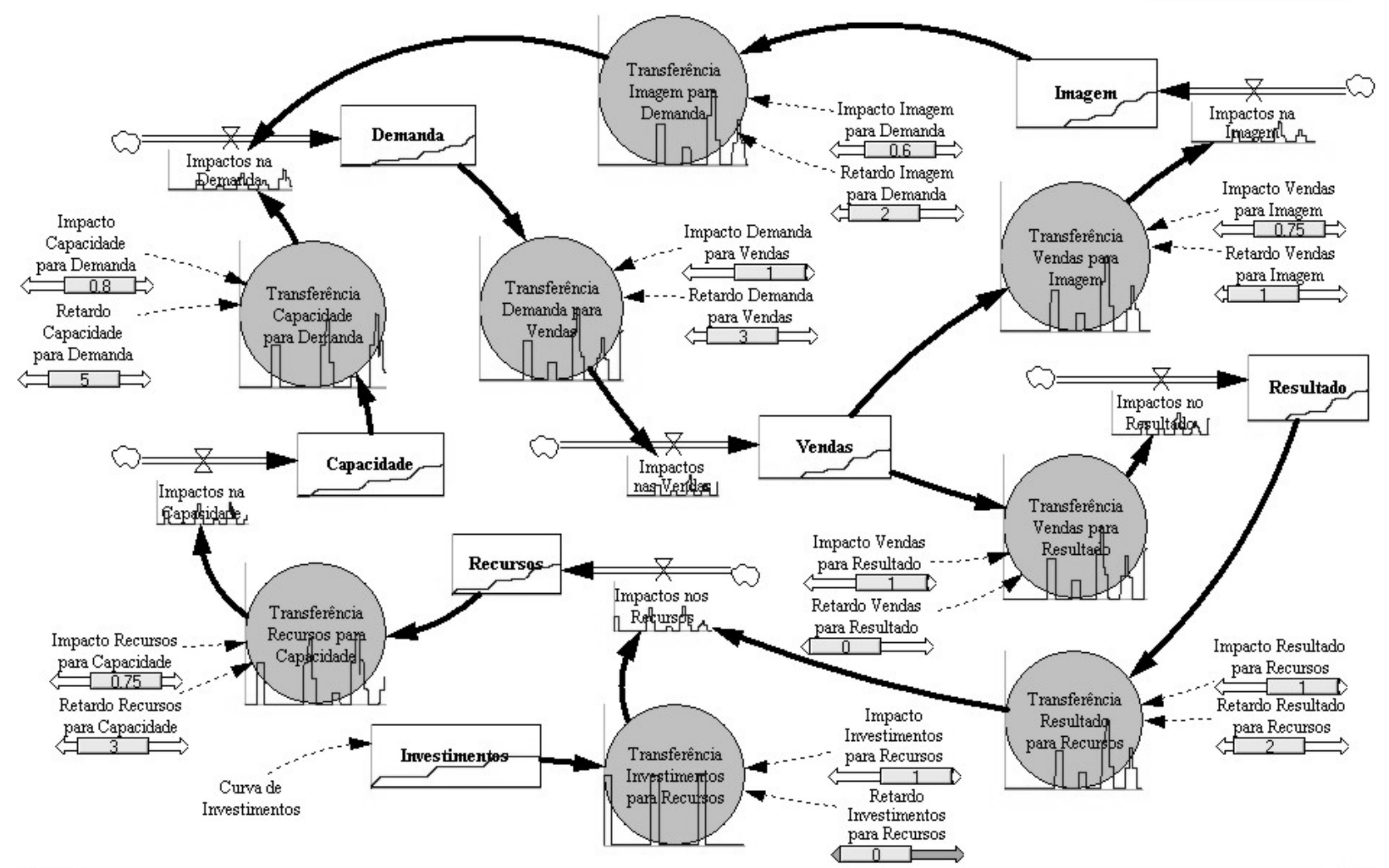

Figura 8: Visão geral do modelo simulado (com tempo de simulação de 36 meses) considerando os valores de impacto e de retardo expostos na Figura 7. Utiliza-se aqui um recurso do software Vensim que permite visualizar o efeito das alterações do modelo em todas as variáveis do sistema.

Fonte: o autor

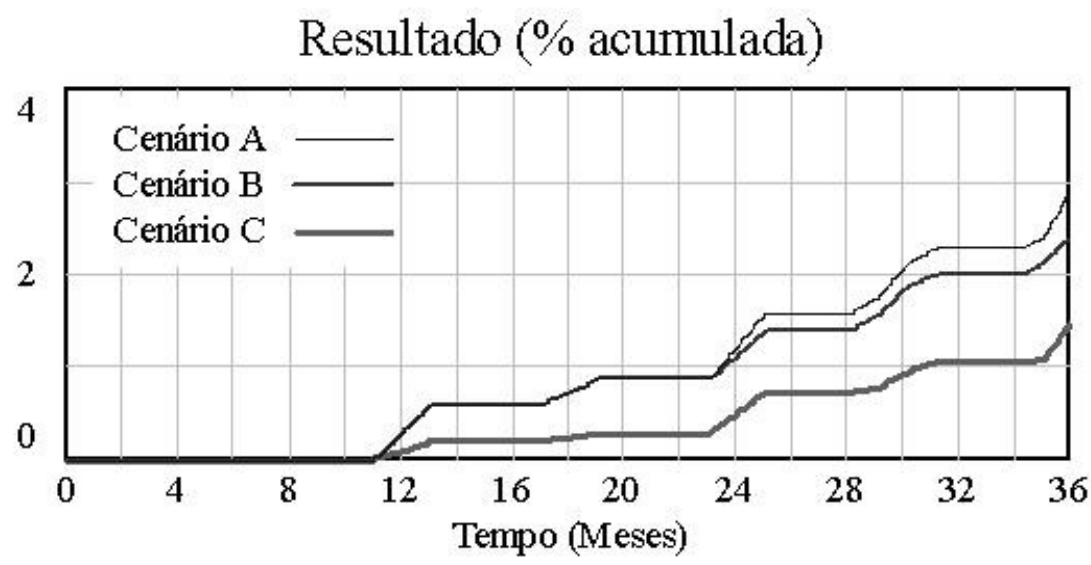

Figura 9: Simulação de 36 meses com os parâmetros da Figura 7 para os cenários A, B e C Fonte: o autor 


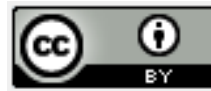

$\mathrm{Na}$ Figura 10 foi mantido o conjunto de parâmetros das simulações feitas anteriormente para os cenários $\mathrm{A}$ e $\mathrm{B}$. Todavia, para a simulação com o cenário $\mathrm{C}$, foi considerado que o impacto dos Recursos sobre a Capacidade aumentaria de 0.75 para 1, isto é, se agora houver um aumento de $100 \%$ nos Recursos, ocorre um aumento de $100 \%$ na Capacidade. Considerou-se também que o tempo de retardo para esse impacto ser sentido cairia de 3 meses para 2 meses.

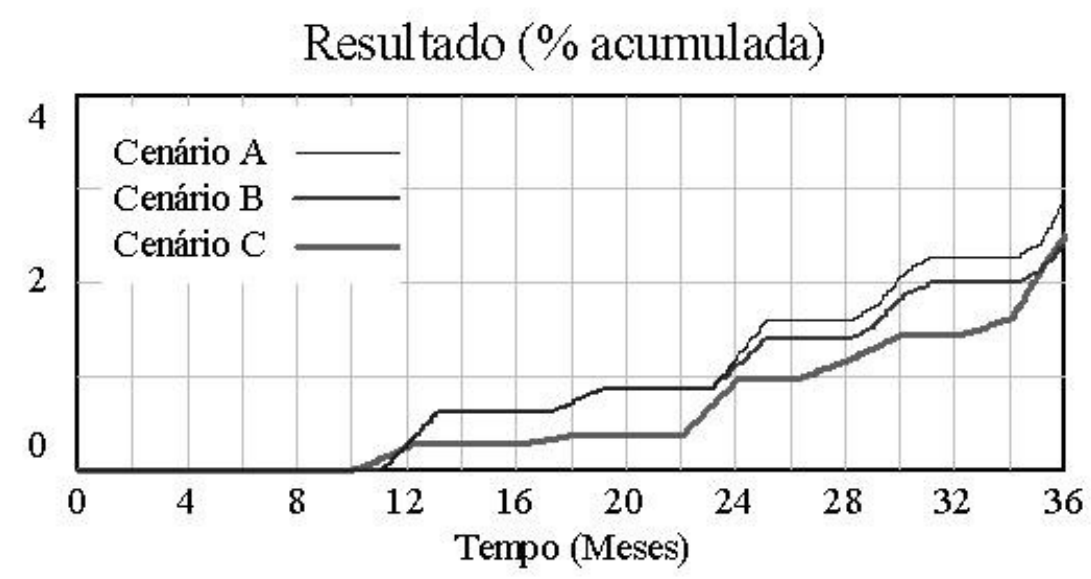

Figura 10: Simulação de 36 meses considerando os parâmetros da Figura 7 para os cenários A e B. Para o cenário C aumentou-se o Impacto de Recursos para Capacidade $(0,75 \rightarrow 1)$ e diminuiu-se o Retardo de Recursos para Capacidade ( $3 \rightarrow 2$ meses).

Fonte: o autor

Obtêm-se então uma curva de Resultado acumulado que se aproxima bem mais das outras duas curvas, chegando a ultrapassar, ao final de 36 meses, a curva gerada pela simulação com cenário B. Esse exemplo ilustra bem o potencial deste tipo de ferramenta para a análise prática de suposições em situação de planejamento.

\section{CONCLUSÕES}

As técnicas de modelagem e simulação têm sido desenvolvidas, em geral, como ferramentas para lidar com situações passíveis de serem matematicamente estruturadas e com uma forte vocação para previsão e otimização. Todavia, a modelagem e a simulação podem e devem ser exploradas também como processos para a captura, a estruturação e a compreensão de nossos modelos mentais e do nosso conhecimento. E é em razão disto, e por ser executada em uma etapa considerada de formulação do problema, dentro do processo de planejamento, 
que a construção de modelos que descrevem o impacto dos cenários no ambiente da empresa deve ser flexível e imaginativa. Permitindo o debate sobre a maioria das suposições a respeito das relações que se tem em mãos.

Essa modelagem não deve consumir tempo ou incomodar. Ela não deve criar uma falta de incentivo para os participantes, devendo ser suportada por ferramentas simples e de fácil manuseio (Senge, 1994, p. 225).

Este trabalho mostrou que é possível atender a esses requisitos. Ele contribui com a construção de um método simples, que oferece àqueles que trabalham com planejamento, principalmente no escopo da análise de cenários e do comportamento sistêmico das empresas, um instrumento eficiente para lidar com a complexidade com a qual os planejadores se deparam todos os dias. Além disso, a simplicidade conceitual do método apresentado permite que ele seja ampliado facilmente para comportar outros tipos de análises, mais abrangentes.

Por exemplo, considerando o caso da Figura 6, suponha que não se pode determinar com certeza o impacto das Vendas para a Imagem nem tampouco o impacto da Imagem para a Demanda. Todavia, se há uma idéia a respeito das faixas dentro das quais estes valores possam estar, pode-se construir várias simulações que considerem tais faixas.

Imagine que o impacto da Vendas para a Demanda esteja localizado dentro do intervalo [0.55; 0.95] e que o impacto da Imagem para a Demanda esteja localizado dentro do intervalo $[0.4 ; 0.8]$. O resultado final da simulação considerando uma distribuição uniforme de valores dentro destes intervalos é vista no gráfico da Figura 11.

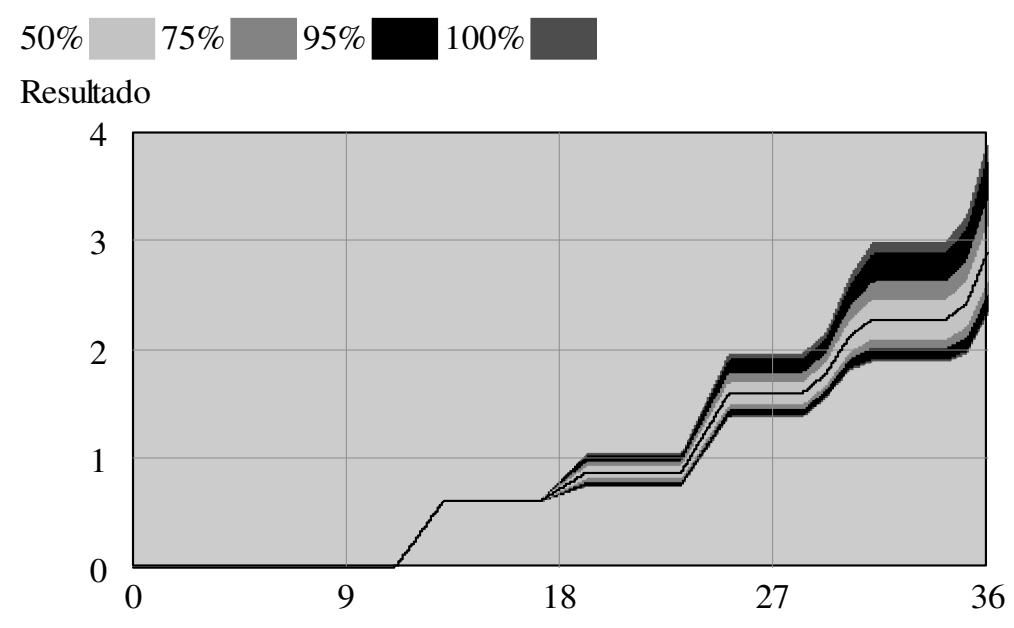

Figura 11: Análise de sensibilidade da variável Resultado. O gráfico é construído variando-se o impacto das Vendas para a Imagem dentro do intervalo [0.55; 0.95] e o impacto da Imagem para a Demanda dentro do intervalo [0.4; 0.8]. A tabela de tons Fonte: o autor corresponde aos intervalos de confiança das variações. 
Em resumo, quando aliadas a formulações matemáticas objetivas e simples, como a apresentada neste trabalho, as técnicas de planejamento orientadas por cenários potencializam sua vocação como ferramentas de aprendizado e criam estímulos para que se testem os limites da nossa realidade.

\section{REFERÊNCIAS}

ACAR, William. Toward a Theory of Problem Formulation and the Planning of Change: Causal Mapping and Dialectical Debate in Situation Formulation. PhD Thesis. University of Pennsylvania: Wharton School, 1983

EDEN, C. Cognitive Mapping. European Journal of Operational Research, 36(1), 1988

FAHEY, Liam e RANDAL, Robert M.; Learning from the Future. New York: John Wiley \& Sons, 1998.

FORRESTER, J. W. Industrial Dynamics. Cambridge: MIT Press, 1961

FORRESTER, J. W. Urban Dynamics. Cambridge: Pegasus Communications, 1969.

GEORGANTZAS, Nicholas e ACAR, William. Scenario-Driven Planning: Learning to Manage Strategic Uncertainty. Westport: Quorum Books, 1995.

HEISE, David R. Causal Analysis. New York: Wiley, 1975

KELLY, George A. The Psychology of Personal Constructs. New York: W. W. Norton, 1955

PIDD, Michael. Modelagem Empresarial: Ferramentas para a tomada de decisão. Porto Alegre: Bookman, 1998.

ROBERTS, N., ANDERSEN, D.F., DEAL, R.M., GARET, M.S. e SHAFFER, W.A. Intoduction to compuer simulation: A system Dynamics modeling approach. Massachusetts: Addison-Wesley, 1983

SENGE, P. M. A Quinta Disciplina: Estratégias e Ferramentas para Construir uma Organização que Aprende - Caderno de Campo. Rio de Janeiro: Qualitymark, 1994.

SHERMAN, Howard e SCHULTZ, Ron. Open Boundaries: Creating Business Innovation Through Complexity. Massachusetts: Perseus Books, 1998.

STERMAN, John D. Business Dynamics: System Thinking and Modeling for a Complex World. Boston: McGraw-Hill, 2000.

VAN DER HEIJDEN, Kees. Planejamento de Cenários. São Paulo: Bookman, 2004. 


\section{Revista Produç@o \\ Associação Brasileira de Engenharia de Produção - ABEPRO Universidade Federal de Santa Catarina - UFSC www.producaoonline.org.br \\ ISSN 1676 - 1901 / Vol. IX/ Num.II / 2009}

VENSIM. Vensim Help Manual. Vensim 5.3a. Disponível em: <http://www.vensim.com>. Acessado em: 28 abr. 2006. 


\section{APENDICE A - Equações do modelo Vensim apresentados neste trabalho}

\section{(Observação: A curva de investimentos corresponde ao cenário A)}

Curva de Investimentos $([(0,0)-(37,5)],(0,0),(1,0.5),(2,1),(12,1),(13,1.5),(14,2),(24,2),(25,2.5),(26,3),(37,3))$

$$
\begin{array}{ll}
\text { Investimentos } & =\text { Curva de Investimentos (TIME) } \\
\text { Demanda } & =\text { INTEG (Impactos na Demanda,0) } \\
\text { Capacidade } & =\text { INTEG (Impactos na Capacidade,0) } \\
\text { Vendas } & =\text { INTEG (Impactos nas Vendas,0) } \\
\text { Resultado } & =\text { INTEG (Impactos no Resultado,0) } \\
\text { Recursos } & =\text { INTEG (Impactos nos Recursos,0) } \\
\text { Imagem } & =\text { INTEG (Impactos na Imagem,0) }
\end{array}
$$

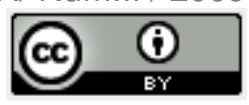

$$
\begin{array}{ll}
\text { Impactos nos Recursos } & =\text { Transferência Investimentos para Recursos+Transferência Resultado para Recursos } \\
\text { Impactos no Resultado } & =\text { Transferência Vendas para Resultado } \\
\text { Impactos nas Vendas } & =\text { Transferência Demanda para Vendas } \\
\text { Impactos na Imagem } & =\text { Transferência Vendas para Imagem } \\
\text { Impactos na Demanda } & =\text { Transferência Imagem para Demanda+Transferência Capacidade para Demanda } \\
\text { Impactos na Capacidade } & =\text { Transferência Recursos para Capacidade }
\end{array}
$$

Transferência Vendas para Resultado=DELAY FIXED(Impacto Vendas para Resultado*(VendasDELAY3I(Vendas, 3*TIME STEP, 0 ))/(3*TIME STEP), Retardo Vendas para Resultado, 0 ) Transferência Vendas para Imagem=DELAY FIXED(Impacto Vendas para Imagem*(VendasDELAY3I(Vendas, 3*TIME STEP, 0 ))/(3*TIME STEP), Retardo Vendas para Imagem, 0 ) Transferência Resultado para Recursos=DELAY FIXED(Impacto Resultado para Recursos*(ResultadoDELAY3I(Resultado, 3*TIME STEP, 0 ))/(3*TIME STEP) ,Retardo Resultado para Recursos, 0 ) Transferência Recursos para Capacidade=DELAY FIXED(Impacto Recursos para Capacidade*(RecursosDELAY3I(Recursos, 3*TIME STEP, 0 ))/(3*TIME STEP) ,Retardo Recursos para Capacidade, 0 ) Transferência Investimentos para Recursos=DELAY FIXED(Impacto Investimentos para Recursos*(Investimentos-DELAY3I(Investimentos, 3*TIME STEP, 0 ))/(3*TIME STEP) ,Retardo Investimentos para Recursos, 0 )

Transferência Imagem para Demanda=DELAY FIXED(Impacto Imagem para Demanda*(ImagemDELAY3I(Imagem, $3 *$ TIME STEP, 0 ))/(3*TIME STEP) ,Retardo Imagem para Demanda, 0 )

Transferência Demanda para Vendas= DELAY FIXED (Impacto Demanda para Vendas*(DemandaDELAY3I(Demanda, $3 *$ TIME STEP, 0 ))/(3*TIME STEP) ,Retardo Demanda para Vendas, 0)

Transferência Capacidade para Demanda=DELAY FIXED(Impacto Capacidade para Demanda*(CapacidadeDELAY3I(Capacidade, 3*TIME STEP, 0 ))/(3*TIME STEP) ,Retardo Capacidade para Demanda, 0 )

$\begin{array}{ll}\text { Impacto Vendas para Resultado } & =1 \\ \text { Impacto Vendas para Imagem } & =0.75 \\ \text { Impacto Resultado para Recursos } & =1 \\ \text { Impacto Recursos para Capacidade } & =0.75 \\ \text { Impacto Investimentos para Recursos } & =1 \\ \text { Impacto Imagem para Demanda } & =0.6 \\ \text { Impacto Demanda para Vendas } & =1 \\ \text { Impacto Capacidade para Demanda } & =0.8 \\ & \\ \text { Retardo Vendas para Resultado } & =0 \\ \text { Retardo Vendas para Imagem } & =1 \\ \text { Retardo Resultado para Recursos } & =6 \\ \text { Retardo Recursos para Capacidade } & =3 \\ \text { Retardo Investimentos para Recursos } & =0 \\ \text { Retardo Imagem para Demanda } & =2 \\ \text { Retardo Demanda para Vendas } & =3 \\ \text { Retardo Capacidade para Demanda } & =5\end{array}$

